\title{
Visual Representation of Text in Web Documents and Its Interpretation
}

\author{
D. Karatzas and A. Antonacopoulos \\ PRImA Group, Department of Computer Science, University of Liverpool \\ Peach Street, Liverpool, L69 7ZF, United Kingdom \\ http://www.csc.liv.ac.uk/ prima
}

\begin{abstract}
This paper examines the uses of text and its representation on Web documents in terms of the challenges in its interpretation. Particular attention is paid to the significant problem of non-uniform representation of text. This non-uniformity is mainly due to the presence of semantically important text in image form as opposed to the standard encoded text. The issues surrounding text representation in Web documents are discussed in the context of colour perception and spatial representation. The characteristics of the representation of text in image form are examined and research towards interpreting these images of text is briefly described.
\end{abstract}

\section{Introduction}

A Web document, like many other types of documents in electronic form, comprises two components: the code and the view. The code, is typically a file containing markup language tags, program instructions and various types of text. To be more precise, text in this instance refers to anything that it is not a keyword or part of a program. This text may not actually appear in the browser window, such as attributes to keywords (e.g. textual attributes to META or ALT tags). On the other hand, the bulk of the text will be visible in the browser window as part of the document text. Typically this text is encoded in ASCII or UNICODE and is formatted for display according to the instructions in the code.

The view of the document is what actually appears in the browser window. This is what humans see when they look at the monitor screen and what the creator of the document intended to present. In the authors' opinion, the view is the definitive representation of the document message as it was originally intended to be conveyed to the reader. The reasons for establishing the view as the baseline representation are explained immediately below.

In a typical Web document, there are significant discrepancies between the text appearing in the view and that in the code of the document. First, text in the code that is due to appear in the browser window may not be visible. This sounds paradoxical but it is true when Web document designers create text in the same colour as that of the background. The rationale in this case, is that search engines will use this (often irrelevant but highly topical) text to boost the rankings of the document in the relevant indices. It should be noted that this approach is an attempt to overcome the fact that some search engines do not index text that is not to be displayed (such as META or ALT tag attributes) [1] as this text is often unreliable (see below). 
A second major (and very frequently occurring) discrepancy is that some of the visible text in the view of the document is actually embedded in images. There is no correspondence between the code (an instruction to display a given image) and the text contained in that image. The human reader, of course, can read all the text on the screen (document view), whether this text is in the code or not. From this point on, visible text that is contained in the code will be referred to as encoded text while text that is embedded in images will be referred to as image text.

The latter discrepancy between the code and the view representations of a Web document is potentially very significant. The origins of the problem are twofold. First, Web document designers create image text as a way of overcoming the limitations of the markup language used in the code. Second, due to limitations of current technology, image text is not accessible to any automated process performed on the document. Both of these interrelated issues are examined next in order to achieve a deeper understanding of the problems of the representation and the impact on the automated interpretation of Web documents.

Image text is created for two main reasons. The first is one of necessity as the markup language (HTML in this case) cannot adequately display textual entities such as mathematical equations, text in diagrams and charts etc. The second and main reason is that document creators wish to add impact to certain textual entities such as titles, headings, buttons etc. The effects applied to the text and its background are such that cannot be expressed in the markup language.

Not having all the visible text in the code of the document means that a proportion of the text seen by the human reader (image text) is not available for any automated analysis. Such analysis includes fundamental processes, fundamental to of the modus operandi of the Web such as automated indexing by search engines. In the case of indexing, the problem is compounded by the fact that it is precisely the semantically important text (titles, headings etc.) that is most often required to make a visual impact and, therefore, represented as image text.

The lack of a uniform representation of the text impacts negatively on several other possibilities for exploiting the Web. If all the visible text was available as encoded text, it would be possible to perform accurate voice browsing [2]. One could listen to the Web document read to them instead of having to look at a monitor. Such a possibility will enable browsing in the car, via the telephone and also will benefit visually impaired people. Another major application area is the analysis of the content of a Web document for filtering, summarisation and display on small form-factor devices such as PDAs and mobile phones.

From the above, it is evident that there is a potentially significant problem of not having a uniform representation of the visible text in a Web document. The remainder of this paper focuses on the problem of achieving such a uniform representation by extracting and recognising the image text. The characteristics of image text are described in Section 2. Image text is usually present in colour (both the foreground and the background). Section 3 briefly discusses the properties of colour and its representation in the context of both the monitor screen and of how humans perceive it (exploited in the authors' approach to extract the image text). Properties of text in terms of its spatial representation in images are presented in Section 4. An overview 
of the challenges faced by current approaches as well as open problems is given in Section 5. The authors' research towards converting image text into its encoded form is summarised in Section 6, while Section 7 concludes the paper.

\section{Image Text Representation}

For encoded text, a pure textual representation exists and is directly available by analysing the code of the web document. In the case of image text, though, information about the textual content of the image is generally absent. The only provision for an alternative representation of image text is the ALT tag, by which a textual description can be supplied for each image. Nevertheless, in an average of $56 \%$ of image text cases, the ALT tag description is incomplete, totally false or inexistent [3]. The same study [3] showed that $76 \%$ of the image text does not appear within the rest of the encoded text. Some information could, potentially, be extracted from the filename of the image, which is usually related to the thematic content of images [4]. However, it can be appreciated that the filename rarely represents an accurate description of the image text.

The ALT tag contents and the filename, along with the size and placement of each image inside a web document, are all the information that can be obtained by analysing the code of the document alone. The other option is to analyse the images themselves, and extract the image text directly from them. As mentioned earlier, this is the most reliable way of obtaining a uniform representation, as the definitive representation is only what the reader sees. Towards this goal, the key characteristics of image text are examined in the remainder this section.

Images found in Web documents share some common characteristics that emanate exactly from the dedicated use of the images in the World Wide Web. Certain observations can be made for image text:

- Image text is created with the use of computers, in order to be viewed on computer monitors.

- File size minimisation is very important when creating image text, as it has to be easily transmitted over the Internet.

- It is used to create impact. Designing eye-catching headers and selection buttons, and enhancing the appearance of a Web document using images for anything that the visitor should pay attention to (e.g., advertisements), is a strong advantage in the continuous effort to attract more visitors.

- There are no strict rules governing the creation of image text e.g., the use of colours, fonts, provision of alternative representation etc. Therefore, people exercise their creativity and frequently produce images with complex colour arrangements of text and background.

Image text presents significant differences from images of scanned paper documents that are typically used by optical character recognition (OCR) applications, which render traditional text extraction methods inapplicable. Certain assumptions that are usually made by OCR applications regarding their input images are not pertinent to image text in Web documents. Differences can be identified with regard to both the structure and the content of images. 


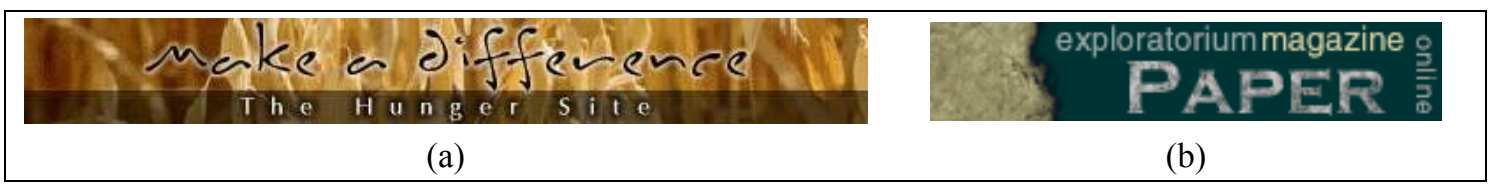

Figure 2-1 - (a) Image containing text over multicoloured photographic background. (b) Image containing multicoloured textured text.

The most prominent difference is the fact that image text is multi-coloured, whereas typical document images are black and white. Traditional scanned document analysis methods that require both the text and the background to be of constant colour are therefore unsuitable for image text in Web documents. The majority of such image text contains gradient-colour or textured characters rendered over textured or photographic background (Figure 2-1). The number of colours present in the image text dataset used by the authors (comprising approximately 120 images of text collected from various Web documents), range from 2 up to 66023 with an average of 4832 colours per image.

Image text is designed to be viewed on computer monitors. This entails certain characteristics with regard to the size and the resolution of the images. Contrary to typical document images, which have a minimum spatial resolution of 300 dpi (dots per inch), characters in image text have an average resolution of $72 \mathrm{dpi}$.

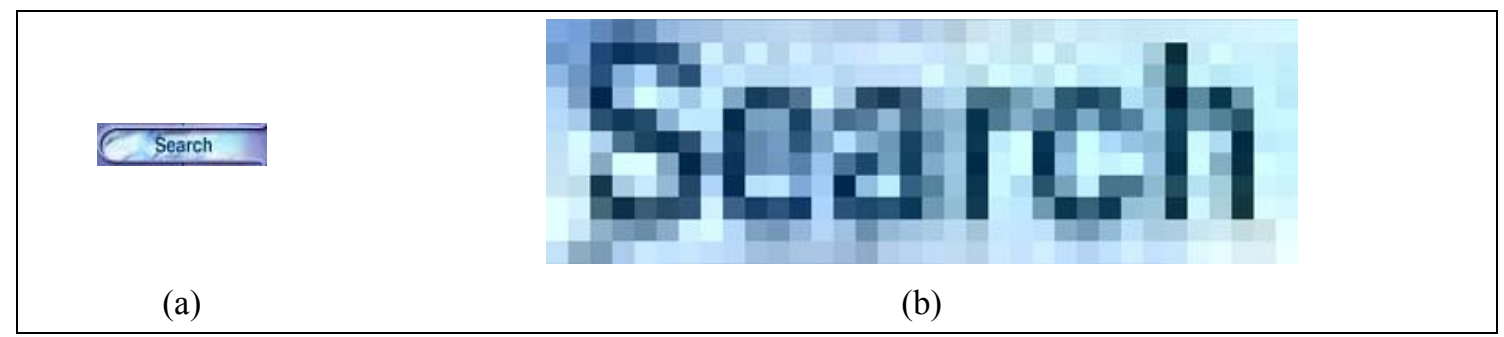

Figure 2-2 - (a) Original image of a menu item. (b) The word "Search" magnified. The effect of antialiasing is severe in this case and, combined with the small size of characters makes this text difficult to recognize, even for humans.

The actual size of the characters is the next significant difference between scanned documents and image text in Web documents. An expected character size in scanned documents is $10 \mathrm{pt}$ or larger, whereas in image text, characters can be as small as the equivalent of $5 \mathrm{pt}$ to $7 \mathrm{pt}$. Commercial OCR methods typically fail for characters of such small size.

Furthermore, although image text does not suffer from the typical distortions and noise introduced during document scanning, different types of artefacts are evident in most of the cases. Anti-aliasing is probably the most common kind of artefact that strongly affects our ability to differentiate characters from the background. Antialiasing is extensively used when rendering text, especially when it comes to smallsized characters, since it produces an aesthetically better outcome. In general terms, it involves a process of blending a foreground object to the background, creating a smooth transition from the colours of one to the colours of the other. This produces characters with poorly defined edges, in contrast to the characters in typical document images (Figure 2-2). Another artefact is that due to the sampling grid used by software 
packages when applying colours to objects, the same character can appear slightly different in different parts of an image.

Finally, the fact that image text is created with file-size minimisation in mind, suggests that most often some kind of compression method is applied to the image. The vast majority of images in the Web are in fact in JPEG format, using in some extent $J P E G$ 's compression capabilities. This type of compression introduces certain artefacts in the images that have no particular effect in areas of almost constant colour, but can produce significant distortions to characters. This kind of lossy compression is even more noticeable when colour analysis of the image takes place, as lightness information is mostly preserved, but colour information is to a great extent discarded in the JPEG compression scheme. The next most popular format used for storing image text, is GIF. Although the GIF format preserves much more information than JPEG, it is limited to representing 256 colours. This vastly reduces the number of available colours to represent the characters with, and can introduce significant quantisation artefacts in the attempt. In addition, due to the limited number of colours available, dithering techniques are often employed to render colours that cannot be represented uniquely (Figure 2-3). Dithered areas are difficult to identify as uniform regions, which poses a further problem in colour image analysis.

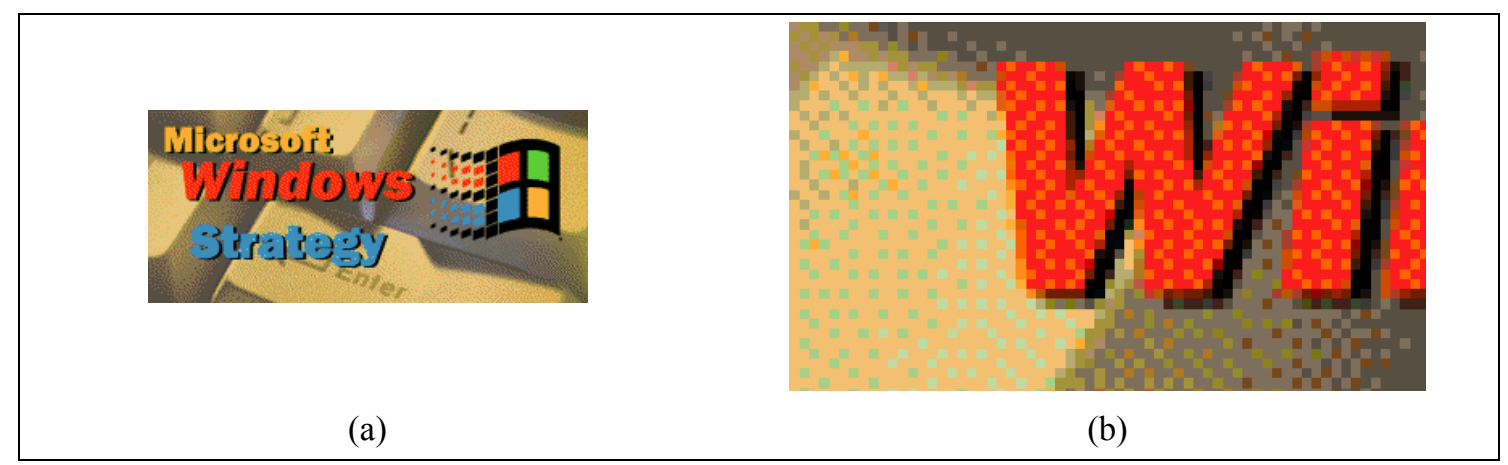

Figure 2-3 - (a) Original GIF compressed image. (b) Magnification of an area containing part of characters and part of the background. Dithering is evident and pixels belonging to the same area would be assigned to different colour clusters in most colour image analysis techniques.

\section{Colour Representation}

Colour is the perceptual result of light in the visible region of the spectrum (having wavelengths between approximately $400 \mathrm{~nm}$ to $700 \mathrm{~nm}$ ). A good understanding of how colour is reproduced on computer monitors and the way it is internally represented in a computer with the use of a colour system is vital to understand the difficulty of analysing colour images such as the ones found in web documents.

Colour is reproduced in Cathode Ray Tube (CRT) displays in an additive manner by mixing three lights of different colours (red, green and blue) produced by the phosphors of the screen. Thus three components are being used, namely $R, G$ and $B$ which express the participating power of each mixing colour. Each component is quantised in $2^{8}=256$ levels, thus a CRT display can produce $256^{3}$ colours, by mixing different amounts of light of each colour. Depending on the technical and physical characteristics of the CRT display, only a certain gamut of colours can be produced. The largest range of colours will be produced with primaries that appear red, green 
and blue, and that is the reason why phosphors producing colour stimulus with exactly those primaries are employed. Nevertheless, since there are hardware differences between computer systems, $R G B$ information alone is not (strictly speaking) adequate to determine the actual colours of an image. A set of primaries that closely represent the primaries used in CRT monitors are the ones specified for the HDTV protocol by the standard ITU-R recommendation BT.709 [5]. The majority of monitors conform to Rec.709 within some tolerance, so it is a safe assumption that the same $R G B$ code will produce the same colour on different CRT monitors.

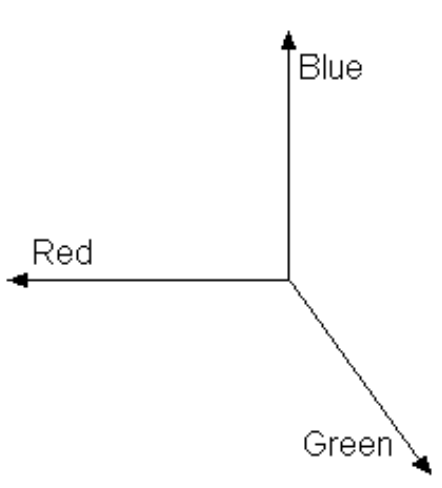

(a)

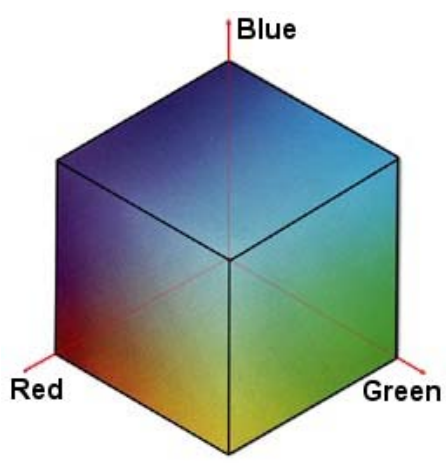

(b)

Figure 3-1 - $R G B$ colour space. (a) Axis of the colour space. (b) Colour gamut.

The most widely used colour system in computer applications is therefore $R G B$ (Figure 3-1). As already mentioned, $R G B$ colour system directly describes the way colours are mixed on computer monitors. Although $R G B$ is hardware dependant, in the sense that the same $R G B$ colour may be slightly different between different monitors, it is the default choice for most applications because of its simplicity and low computational cost.

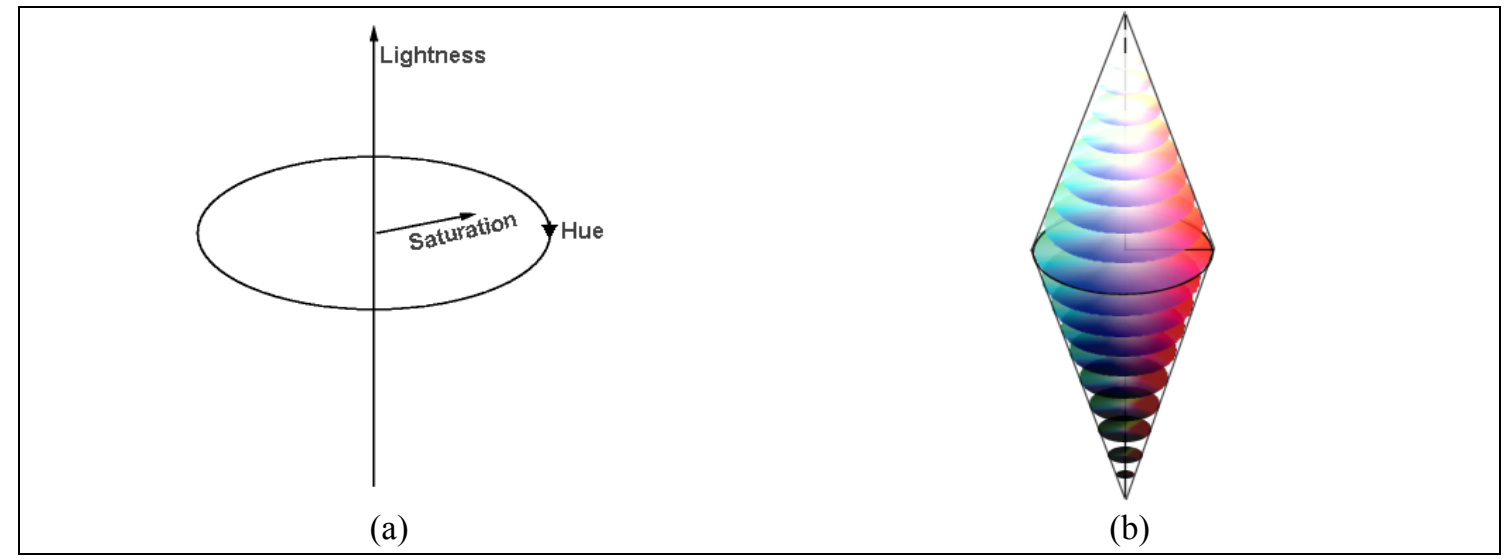

Figure 3-2 - HLS colour space. (a) Axis of the colour space. (b) Colour gamut.

A number of colour attributes can be calculated from the $R G B$ components. An interesting set of attributes, in the sense that they are representative of human perception, is Hue, Lightness and Saturation. These are psychological attributes related to human impressions of colour. The use of such perceptually based quantities can prove more suitable for the analysis of images created to be viewed by humans 
such as real-life scenes, and for this case, image text [6, 7]. HLS, HVC and HSI are colour systems based on these attributes (Figure 3-2).

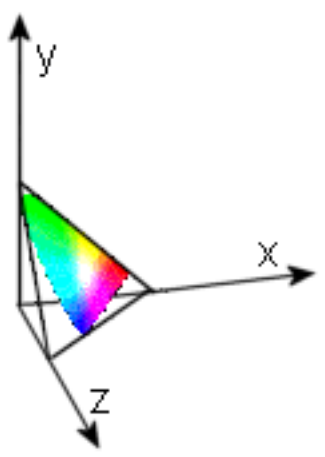

(a)

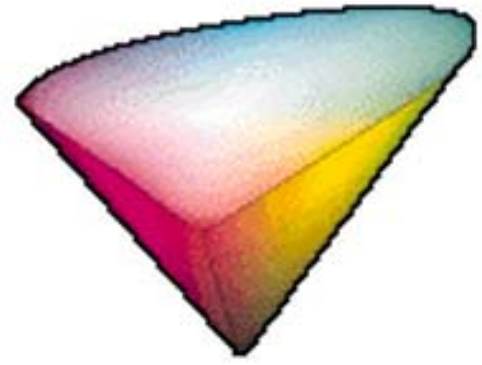

(b)

Figure 3-3 - CIE $X Y Z$ colour space. (a) Axis of the colour space. (b) Colour gamut.

There exists, however, a totally different approach based directly on human vision characteristics rather than on transformations of the $R G B$ components. A colour stimulus is radiant energy of given intensity and spectral composition, entering the eye and producing a sensation of colour. This radiant energy can be completely described by its spectral power distribution. This is often expressed in 31 components, each representing power in a $10 \mathrm{~nm}$ band from $400 \mathrm{~nm}$ to $700 \mathrm{~nm}$. Using 31 components is a rather impractical and inefficient way to describe a colour, especially when a number of colours must be described and communicated, which is the case with computer graphics. A more efficient way would be to determine a number of appropriate spectral weighting functions to describe a colour, and it proves that just three components are adequate for that, based on the trichromatic nature of vision. The CIE (Commission Internationale de l'Eclairage or International Commission on Illumination) standardized, in 1931, a set of spectral weighting functions, called Colour Matching Functions, which model the perception of colour by human beings. These curves are referred to as $\bar{x}, \bar{y}$, and $\bar{z}$, and the colour system subsequently defined as CIE $X Y Z$ (Figure 3-3).

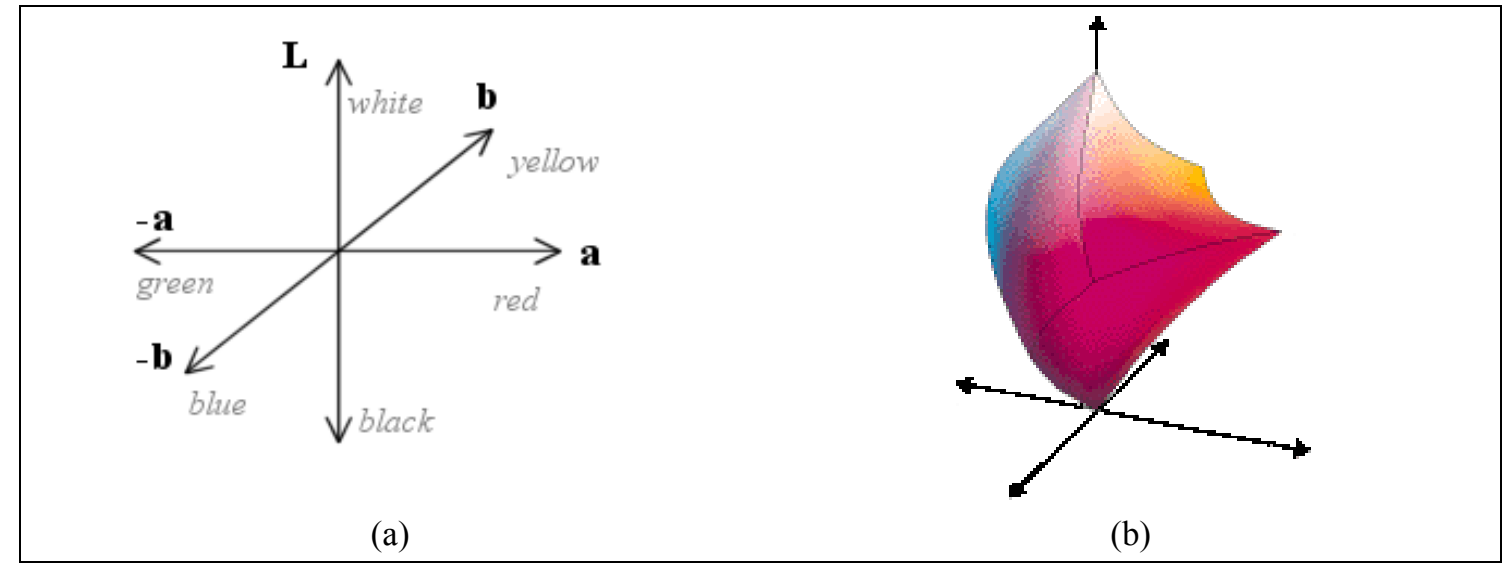

Figure 3-4 - CIE $L^{*} a^{*} b^{*}$ colour space. (a) Axis of the colour space. (b) Colour gamut.

A significant problem with most colour systems (including XYZ) is that the distance between two colours in the colour space does not correlate in any way with the 
perceived (by humans) distance of the colours (how similar or dissimilar they are). For this reason, the CIE proposed certain transformations of the XYZ colour system, resulting in systems that exhibit greater perceptual uniformity. The CIE $L^{*} a^{*} b^{*}[8,9]$ and CIE $L^{*} u^{*} v^{*}[10]$ are such colour systems (Figure 3-4), and are used when a colour distance measure that correlates well to the perceptual colour distance is needed.

\section{Spatial Representation}

Although colour information is vital when trying to separate the foreground from the background of an image, there is an additional number of spatial characteristics that enable us to infer whether something in the image is a character, even if it is a character we have never encountered before. In this section, the spatial features of characters indicative of their hypostasis are briefly summarized in the context of image text.

A distinctive feature of characters is the fact that they are comprised of strokes. A stroke can be thought of as a single movement of the writing tool. In the context of image text, a stroke can be any short line, straight or curved, which is part of a character. All characters can be decomposed to a series of strokes as can be seen in figure Figure 4-1. This is an important observation, since it directly suggests a way to create a comprehensive description of every character. Such a description can be obtained by recording the character's strokes and the way they are combined, in terms of corners, ends and intersections. Descriptions like the above are invariant of size and in most of the times of rotation, and are widely used in character recognition applications. Although such a matching process usually comes after segmentation, that is after the characters have been separated from the background, the knowledge that characters comprise from a number of strokes can provide useful information for the segmentation process as well.

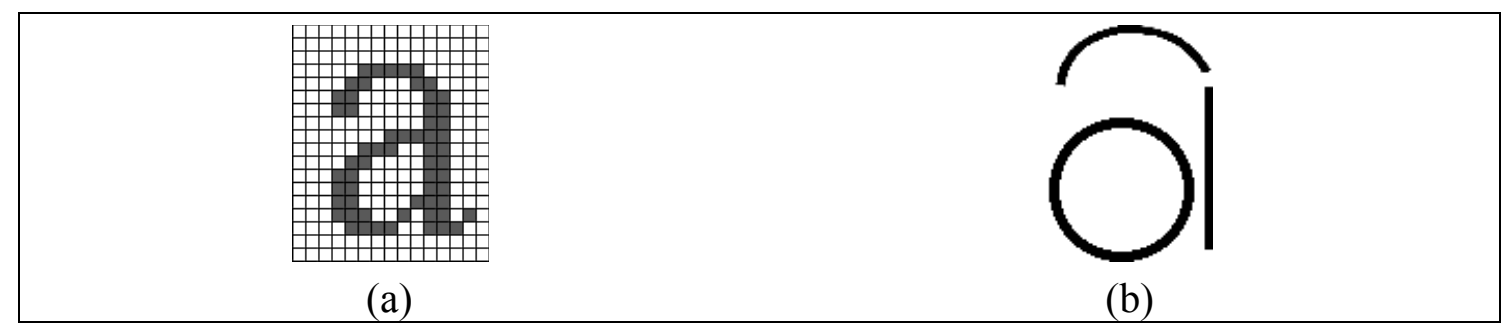

Figure 4-1 - (a) Original character. (b) The character decomposed in a number of strokes: an arc, a straight line and a circle.

A second prominent feature of characters is their aspect ratio. This is defined as the ratio of the character's width to its height or vice versa, and is a measure of the overall shape of the character (in terms of elongated its bounding box is). In general, with the exception of characters like "i" or "l", the bounding boxes of characters are closer to square, with an aspect ratio near 1 .

Other spatial features that can be used towards the identification of characters in images are the percentage of the area of a character's bounding box occupied by character pixels (as opposed to pixels describing the background), and the number of transitions from character pixels to background ones and vice versa within the bounding box of the character. 


\section{DISNEP.COI}

(a)

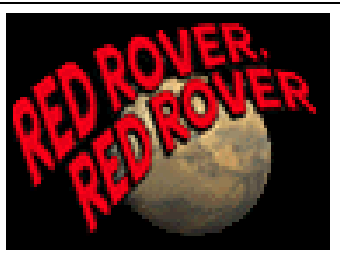

(b)

Figure 4-2 - (a) Image with characters of different font in the same line of text. What is also interesting is that part of the first character is missing (placed in a different image in the web document). This is an example of tightly cropping the images around the characters, even splitting characters among different images. (b) Characters of the same line of text are of different size. Also they are not placed on a straight line as usually the case in paper documents.

At a more macroscopic scale, when looking at the whole set of characters in an image or document, we usually expect them to share some common characteristics. The size of characters is probably the first such characteristic. Indeed, in the majority of the cases we expect the characters in a paragraph, or at least within a single line of text to have similar size. Although such assumptions stand true for essentially all paper documents, when it comes to image text the situation is relatively different. There are many cases where even characters of the same word are of different font and consequently of different size as well (Figure 4-2). To make things even worse, there are cases where characters are substituted by other shapes for the sake of visual impact, as can be seen in Figure 4-3.

\section{LYCOS Your Personal Internet Guide}

Figure 4-3 - Example of an image where characters have been replaced by other shapes.

At the image level, one could study features such as the proportion of foreground pixels to background ones for the whole image. Essentially what part of the image is occupied by characters, which is the foreground class in this case. Knowledge of the expected coverage of the image by characters could prove useful in the process of selecting the foreground colour class for the image. In simple cases where both the text and the background are of constant colour, the selection of the colour corresponding to characters could be initially based on exactly this kind of information. Of course, this simplified case only applies to images such as scanned documents and certainly not in multi-coloured image text in Web documents. Nevertheless, one could use this information to evaluate the final segmentation achieved by other methods, and evaluate subsequently whether the classes are identified as expected. Still, the special use of images on the web does not allow for such an approach, since there are numerous images that do not contain full paragraphs, but only single words or, even worse, single characters as parts of a larger set. Furthermore, characters in image text are often cropped tightly around their outlines (Figure 4-2a), and they have no equivalent to the white frame, present in document images, thus the proportion of the image area occupied by characters varies significantly. 
Finally, an important characteristic of text is that characters are usually placed on straight (and horizontal) baselines. While this is true for the majority of paper documents, characters in image text in Web documents may not be on straight or even horizontal baselines (as depicted in Figure 4-4).

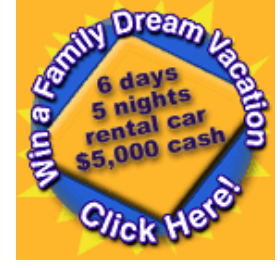

(a)

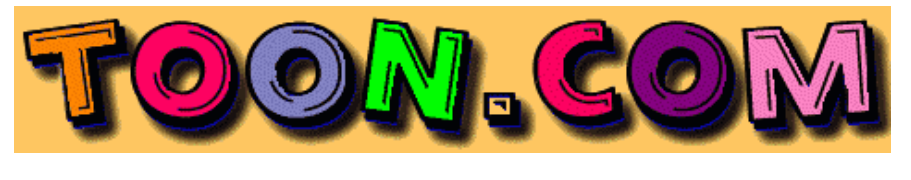

(b)

Figure 4-4 - (a) Sentences placed on a circle, and straight lines of text placed on an angle. (b) Letters of the same 'word' placed with different orientation.

Overall, concerning the spatial characteristics of text as a whole or of single characters, their applicability to image text in Web documents proves more limited compared to traditional scanned documents. However, in combination with other features, such as colour similarity, spatial characteristics can provide considerable help in numerous circumstances.

\section{Challenges and Approaches}

The characteristics of the image text have been examined in the previous sections in terms of image, colour and spatial representations. This section examines the problem of extracting the characters from the image text and the subsequent recognition of this text. As the character extraction is still an unsolved and difficult problem and there is only one approach in the literature that has attempted to recognise the characters, this section concentrates on the former.

A small number of approaches have been proposed towards text extraction from image text. Previous attempts, mainly assume that the characters are of uniform colour, work with a relatively small number of colours, and restrict their operations on the RGB colour space.

One of the most prominent approaches is that of Zhou and Lopresti [11-13]. They proposed methods for both text extraction and recognition. The images used are GIF formatted (256 colours only), and the characters are assumed to be rendered in a homogeneous colour. Their method for text extraction is based on clustering in the RGB colour space, and subsequently identifying connected components in the image according to the clusters located. A detection rate of $47 \%$ was initially reported for a data set comprised by GIF images collected from the Web. An optimisation of the algorithm was later proposed $[14,15]$ which introduced a metric that combines RGB Euclidean distance with the spatial proximity of pixels having the same colour computed in a small neighbourhood. The definition of such a metric is feasible since the images used are only GIF formatted, thus they contain a maximum of 256 colours. A layout analysis follows the connected component identification stage, which aims to identify the character-like components based on spatial features of text, making certain assumptions for the placement of characters. They report an average character 
detection rate of $68.3 \%$ for a set of 482 GIF images collected from the Web, containing homogeneous text.

With similar assumptions about the colour of characters, the approach of Antonacopoulos and Delporte [16] uses two alternative clustering approaches in the RGB space, but works on (bit-reduced) full-colour images (JPEG) as well as GIFs. Jain and $\mathrm{Yu}$ [17] report a method based on decomposing an original image into a number of foreground images and a single background one. The original number of colours (8-bit or 24-bit images) is dramatically reduced (to between 4 and 8 distinct colours) by bit dropping and colour quantisation in the RGB space.

\section{An Anthropocentric Approach}

Towards the extraction of characters from image text, the authors have examined possible ways to segment and identify character-like components in colour images. Two different methods have been implemented and tested. The innovation of both approaches, lies to fact that they are both based on available knowledge on the way humans perceive the given images. The anthropocentric character of the two approaches is evident primarily through the way colour is manipulated, making use of human perception data and employing colour systems that are efficient approximations of the psychophysical manner humans understand colour.

The first method proposed by the authors [18] works on a split and merge strategy. It employs the HLS colour space to split the image into layers in a recursive way, by analysing the Luminance and the Hue histograms. Connected components are then identified, and for each component, the neighbouring pixels are checked for colour similarity. In this way, a visually similar area is identified for each component as a possible extension. Special consideration has been given to the way visual similarity is assessed. Towards that end, the authors used existing biological data for wavelength and luminance discrimination, according to the layer processed each time. The merging process starts with the bottom layers and proceeds in a bottom-up manner. The merging of components (and their possible extensions) is ruled by the extent to which they overlap.

The second method developed by the authors [19], is based on the use of a propinquity measure defined with the help of a fuzzy inference system. The method comprises two steps. It starts with the grouping of pixels having similar colours into connected components, and then uses the propinquity measure defined, to combine connected components into larger ones, aiming at constructing a correct segmentation of the characters in the image. This time the perceptually uniform CIE $L^{*} a^{*} b^{*}$ colour space was used in order to assess the colour similarity of the pixels. The propinquity measure used in the second step combines, with the help of a fuzzy inference system, the colour distance between two components and a metric indicative of their spatial distance. The colour distance metric used is the Euclidean distance in the CIE $L^{*} a^{*} b^{*}$ colour space, while the second input of the fuzzy system is a topological measure defined by the authors, which has to do with the way components are connected in the image.

Both methods were able to correctly segment an average of $60 \%$ of the characters in images containing multicolour-text over multi-colour background. In simpler images, where either the text or the background was mostly uniform, both methods correctly 
segmented around $80 \%$ of the characters. Figure $6-1$, illustrates in a comparative way the resulting segmentation from both methods. Correctly segmented characters are illustrated in black colour, while in red colour are characters that were still correctly separated from the background, albeit not as single, whole characters (broken in more than one components, or joined together).
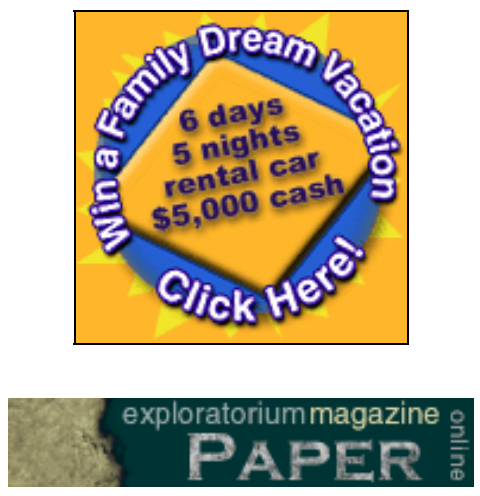

(a)
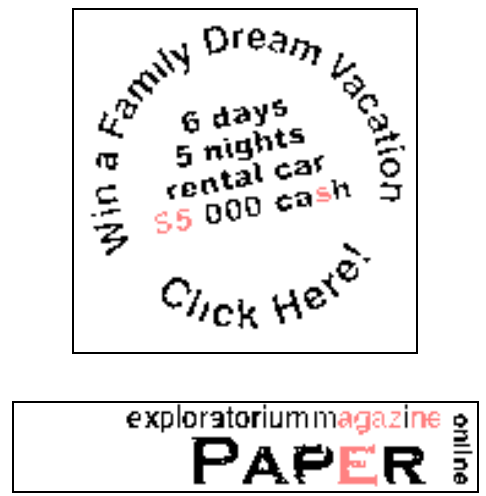

(b)

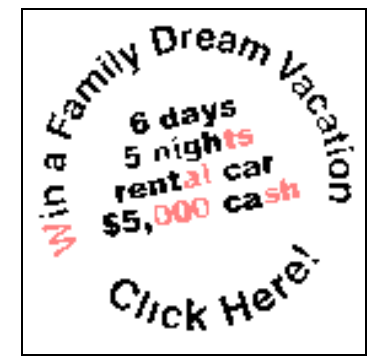

expl rato Ium maqazine

Figure 6-1 - (a) Original image. (b) Results obtained with split and merge method. (c) Results obtained with fuzzy segmentation method..

\section{Concluding Remarks}

From the preceding sections, it can be appreciated that there is a problem of nonuniform representation (in terms of encoding) of text in Web documents. There is a pressing need to do so to achieve more accurate searching and retrieval of information from the Internet. Moreover, there is an ever-increasing requirement to provide the capability for novel ways of interaction with the Internet (e.g., voice browsing and viewing summarised documents on devices with small bandwidth and form-factor).

To obtain a uniform representation for the text in Web documents, the image text must be analysed, the characters within it extracted and recognised. It is evident that the various characteristics if the representation of the image text (image, colour and spatial representation) make the interpretation of image text a difficult problem.

Finally, the paper described a handful of prominent approaches to interpret the image text (mostly extract the characters from the images at this stage). Among them, the research carried out by the authors attempts to exploit human perception of colour differences along with spatial features of characters. The results obtained so far are very promising and further development of the extraction and subsequent recognition methods is planned for the near future.

\section{References}

[1] "Search Engine Watch," http://searchenginewatch.com

[2] M. K. Brown, S. C. Glinski, and B. C. Schmult, "Web Page Analysis for Voice Browsing," Proceedings of the $1^{\text {st }}$ International Workshop on Web Document Analysis (WDA2001), Seattle, USA, 2001.

[3] A. Antonacopoulos, D. Karatzas, and J. Ortiz-Lopez, "Accessing Textual Information Embedded in Internet Images," Proceedings of the SPIE Internet Imaging II, San Jose, USA, pp. 198-205, 2001. 
[4] E. V. Munson and Y. Tsymbalenko, "To Search for Images on the Web, Look at the Text, Then Look at The Images," Proceedings of the $1^{\text {st }}$ International Workshop on Web Document Analysis (WDA2001), Seattle, USA, 2001.

[5] "Basic Parameter Values for the HDTV Standard for the Studio and for International Programme Exchange," ITU-R Recommendation BT.709 [formely CCIR Rec.709] Geneva, Switzerland: ITU 1990.

[6] R. S. Ledley, M. Buas, and T. J. Golab, "Fundamentals of True-Color Image Processing," Proceedings of the $10^{\text {th }}$ International Conference on Pattern Recognition, pp. 791-795, 1990.

[7] S. Tominaga, "Color Image Segmentation Using Three Perceptual Attributes," Proceedings of the Conference on Computer Vision and Pattern Recognition, pp. 628-630, 1986.

[8] K. McLaren, "The Development of the CIE 1976 (L"a $\left.{ }^{*} b^{*}\right)$ Uniform Colour Space and Colour-difference Formula," Journal of the Society of Dyers and Colorists, vol. 92, pp. 338-341, 1976.

[9] A. L. Robertson, "The CIE 1976 Color Difference Formulae," Color Research and Application, vol. 2, pp. 7-11, 1977.

[10]R. C. Carter and E. C. Carter, "CIE L u v ${ }^{*}$ Color-Difference Equations for SelfLuminous Displays," Color Research and Application, vol. 8, pp. 252-253, 1983.

[11]J. Zhou, D. Lopresti, and Z. Lei, "OCR for World Wide Web Images," Proceedings of the IS\&T/SPIE International Symposium on Electronic Imaging, San Jose, California, pp. 58-66, 1997.

[12]D. Lopresti and J. Zhou, "Document Analysis and the World Wide Web," Proceedings of the Workshop on Document Analysis Systems, Marven, Pennsylvania, pp. 417-424, 1996.

[13] J. Zhou and D. Lopresti, "Extracting Text from WWW Images," proceedings of the $4^{\text {th }}$ International Conference on Document Analysis and Recognition, Ulm, Germany, 1997.

[14]D. Lopresti and J. Zhou, "Locating and Recognizing Text in WWW Images," Information Retrieval, vol. 2, pp. 177-206, 2000.

[15]J. Zhou, D. Lopresti, and T. Tasdizen, "Finding Text in Color Images", Proceedings of the IS\&T/SPIE Symposium on Electronic Imaging, San Jose, California, pp. 130-140, 1998.

[16]A. Antonacopoulos and F. Delporte, "Automated Interpretation of Visual Representations: Extracting Textual Information from WWW Images," in Visual Representations and Interpretations, R. Paton and I. Neilson, Eds. London: Springer, 1999.

[17] A. K. Jain and B. Yu, "Automatic Text Location in Images and Video Frames," Pattern Recognition, vol. 31, pp. 2055-2076, 1998.

[18] A. Antonacopoulos and D. Karatzas, "An Anthropocentric Approach to Text Extraction from WWW Images," Proceedings of the the $4^{\text {th }}$ IARP Workshop on Document Analysis Systems (DAS2000), Rio de Janeiro, Brazil, pp. 515-526, 2000.

[19]A. Antonacopoulos and D. Karatzas, "Text Extraction from Web Images Based on Human Perception and Fuzzy Inference," Proceedings of the $1^{\text {st }}$ International Workshop on Web Document Analysis (WDA2001), Seattle, USA, pp. 35-38, 2001. 